International Journal of Intelligent Communication, Computing and Networks Open Access Journal (ISSN: 2582-7707)

https://doi.org/10.51735/ijiccn/001/21

\title{
Approach to Avoid Resource Exhaustion Caused by Editing Tools for Automating Effects Using Noise Inducing Procedures in Deep Learning
}

\author{
Raunak M. Joshi ${ }^{a}$, Dr. Deven Shah ${ }^{b}$ \\ Thakur College of Engineering and Technology, Mumbai, India ${ }^{\mathrm{a}-\mathrm{b}}$ \\ raunakjoshi.m@gmail.com ${ }^{\mathrm{a}}$, sir.deven@gmail.com ${ }^{\mathrm{b}}$
}

\begin{abstract}
Editing softwares are extensively exhausts the resources in terms of hardware, software and manpower. The resource allocation requires collective gathering of funding. Since the last many years Deep Learning has proven to handle and automate heavy multiprocessing tasks very efficiently with less resource exhaustion. It requires resources for training in the first phase and later just uses stored weights of the model resulting in less resource allocation. In this paper we propose an idea that can leverage the power of Deep Neural Networks for Image Processing applications pertaining to Deep Learning for automating blur effects provided by editing software. Our approach revolves around the idea of refactoring Yolact, Real Time Object Detection and Image Segmentation Network Bounding Boxes, Probabilities and Masks with Gaussian Noise Inducing Procedure for making the desired changes in objects.
\end{abstract}

Keywords: Deep Learning, Object Detection, Image Segmentation, Gaussian Noise, Yolact

\section{Introduction}

Deep Learning has proven to be successful in the area of Image Processing Applications. Deep Learning [1] is a non-parametric predictive approach and composes aspects of Inferential Statistics as its base structure. It is a blend of mathematical areas such as Statistics, Linear Algebra and Calculus. Deep Learning is performed using an Artificial Neural Network [2] which is derived from actual human brain neuron structure. With a rise in the amount of data, these networks have become Dense over the period of time. Dense networks have more activation neurons. More the number of neurons, higher is the possibility of learning parameters. This sometimes assists a better learning curve for the network. The applications of Image Processing in Deep Learning are solved using Convolutional Neural Network $[3,4,5]$.

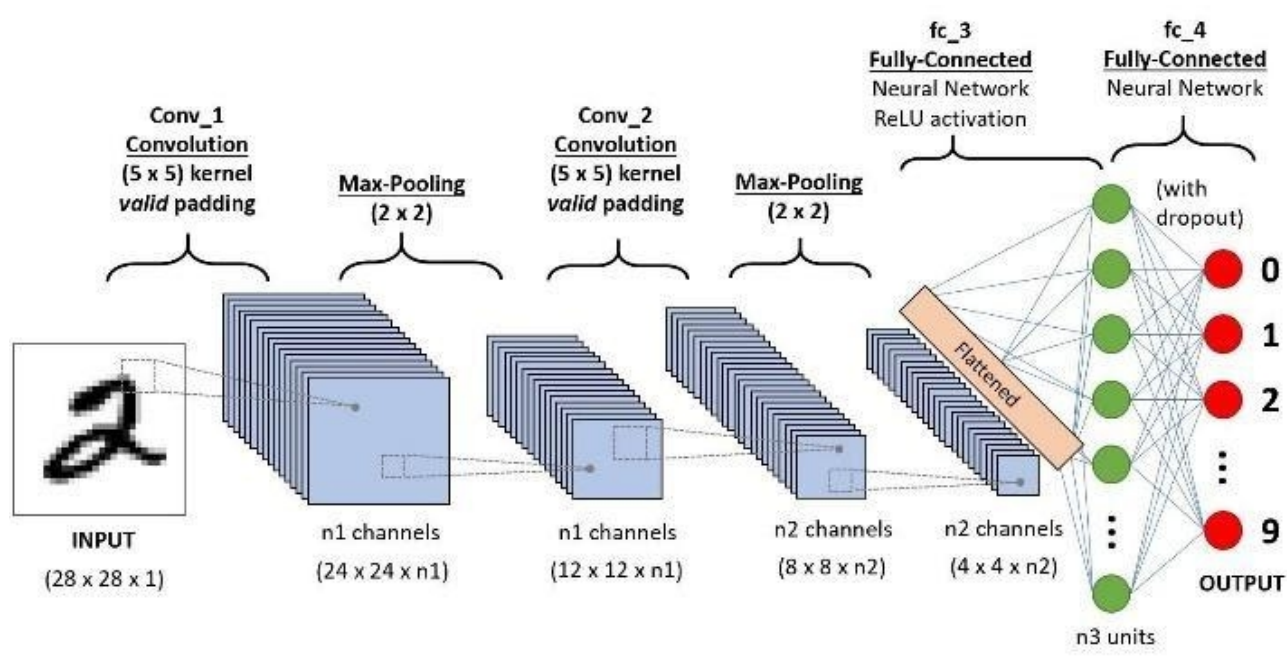

Figure 1: Convolution Neural Network (Source : Sumit Saha - A Comprehensive Guide to Convolutional Neural Networks - the ELI5 way) 
International Journal of Intelligent Communication, Computing and Networks

Open Access Journal (ISSN: 2582-7707)

https://doi.org/10.51735/ijiccn/001/21

The method of Convolutional Neural Network works with a fully functional neural network that takes the input as images and gives desired output. The convolutional neural network uses the approach of convolutions which is a mechanism designed to capture the features from an input image. The features extracted from the image are done using the edge detection. The edges are not just horizontal or vertical, but in many directions. This feature extraction is done using filters. These filters are also known as kernels or feature extractors. These filters play an important role in convolution operation. These filters are applied on the image and they collect the features from the image. Filters are particularly designed to perform certain operations. The convolution operation picks up the features from every single pixel value. This operation takes the value of the filter's pixel and value of the input image's pixel and performs a multiplication operation on it. Similarly it takes the pixel value of every single pixel and adds them to get the value of the pixel of the output filter. The question arises is the size of the output filter dimension. There is an equation for it.

$$
\frac{\text { DImension of the image }- \text { Dimension of filter }}{\text { Strides taken }}+1
$$

Assuming an example where the dimension of the image is $28 \times 28$ pixels and filters is $3 \times 3$ which takes stride of 1 , the size of output will be $26 \times 26$. There are also a number of channels which determine the depth of the neural network. Assuming we use a set of 64 filters which collect different types of information from the image gives the dimension of $26 \times 26 \times 64$. The size of activation neurons in this operation is around 43,264 since $26 \times 26 \times 64$. If the input layer is a grayscale image then the amount of parameters learnt in this layer are 640. These parameters are calculated with the help of an equation. Considering the equation given below $(((3 \times 3 \times 1)+1) * 64)$ we get 640 . In the equation given below $\mathrm{H}$ is height of filter, $\mathrm{W}$ is width of filter, FP is number of filters in the previous layer, 1 is for bias and FC is number of filters in the previous layer.

$$
(((H * W * F P)+1) * F C)
$$

The convolution layer is not enough. The entire procedure requires a Pooling layer to be called as one layer that extracts features. The function of Pooling Layer is to take the elements that are necessary from the convolved image. It is a matrix with specified dimension that takes strides over the convolved image and picks the highest number from the matrix. The size can be determined with a formula same as the convolution dimension estimation.

$$
\frac{\text { Dimension of the convolved image }- \text { Dimension of Pooling filter }}{\text { Strides taken }}+1
$$

Multiple layers can be used. In the final layers, the converged image features are flattened to form a long chain vector of values. These proceed further in the form of Fully Connected Layers. These layers are standard stacked neurons in a dense fashion. These layers are connected with each other in specific fashion with activation functions just like the standard neural network. The way we can calculate the parameters for these layers is the same as the Deep Neural Network. In the equation given below NC is Neurons in Current Layer and NP is Neurons in Previous Layer.

$$
((N C * N P)+(1 * N C))
$$

\section{Review}


International Journal of Intelligent Communication, Computing and Networks Open Access Journal (ISSN: 2582-7707)

https://doi.org/10.51735/ijiccn/001/21

In 2014, Olga Russakovsky, Jia Deng, Hao Su, Jonathan Krause, Sanjeev Satheesh, Sean Ma, Zhiheng Huang, Andrej Karpathy, Aditya Khosla, Michael Bernstein, Alexander C. Berg, Li Fei-Fei proposed the ImageNet Large Scale Visual Recognition Challenge [6]. That project was a powerful benchmark setter. ImageNet is an extremely massive dataset that requires higher computational ability. It has over 1000 classes. The challenge was later posted for everyone to find the most optimal solution. The challenge was extensively exhaustive 5 years' worth work with many contributors. The challenge was annually started from 2010 with stellar background people.

In 2015, Joseph Redmon, Santosh Divvala, Ross Girshick, Ali Farhadi proposed YOLO [7], a new approach to object detection. YOLO is much like a Fully Convolutional Neural Network also abbreviated as FCNN. It works with the Bounding Box principle. These bounding boxes are a set of grids that are mapped on entire frames of videos or entire images. The bounding boxes have class specific confidence scores for every single box in its prediction mechanism. Standard YOLO was trained with 24 conv layers followed by 2 fully connected layers which makes it an extremely mature neural network. There are labels for every single of the objects detected in the output vector. These are sequentially arranged and the network calculates probabilities for every classified class and gives confidence which is then considered as the output pertaining to desired thresholds. It uses the concept of Intersection Over Union which helps users classify multiple objects in respective bounding boxes.

\section{Methodology}

The first approach we gave a thought was obviously using the Object Detection Method designed specifically for Deep Learning. Object Detection was a good start as we wanted something more precise that can detect faces from videos or images. The object detection works on the mechanics of the bounding boxes. These boxes are able to detect the objects with cascades. You Only Look Once abbreviated as YOLO [7] is a high dimensional object detection algorithm created for deep learning object detection. The bounding boxes scavenge for objects in every single frame. The overlapping boxes are recognized by Intersection over Union (IoU). YOLO was an appropriate starting algorithm for our idea as we would detect the faces in our video frames.
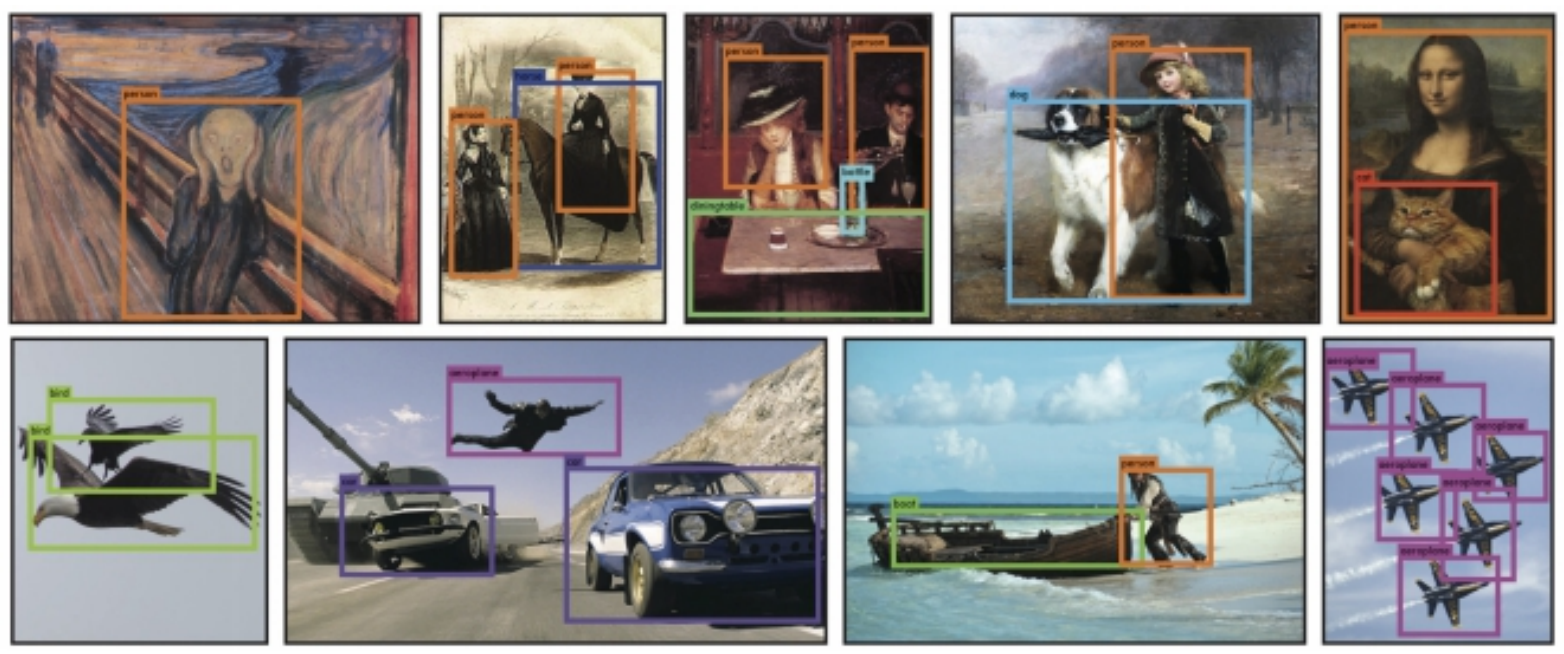

Figure 2 : YOLO Object Detection Network (Source : You Only Look Once: Unified, Real-Time Object Detection, 2016 IEEE Conference on Computer Vision and Pattern Recognition (CVPR))

Object Detection only works for Detection but our idea wanted something more accurate. Image Segmentation was a better approach as we wanted to extract specific features from the input. Under 
International Journal of Intelligent Communication, Computing and Networks Open Access Journal (ISSN: 2582-7707)

https://doi.org/10.51735/ijiccn/001/21

this contention we started our search along with autoencoders and we came across U-Net [8]. It was designed for Biomedical Image Segmentation and was very powerful. It is a very dense convolutional autoencoder. It works on the skip connections concept and has over 30 million parameters.
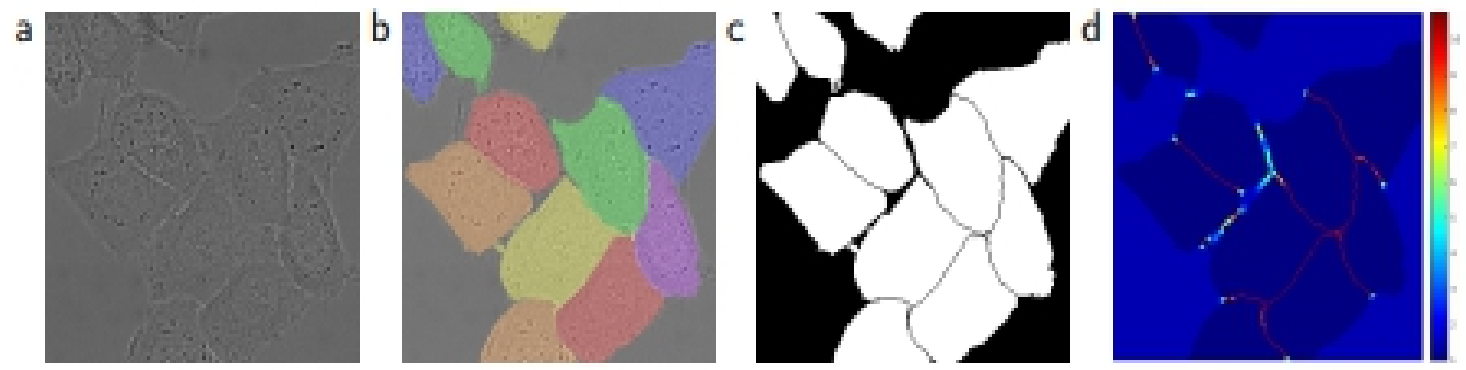

Figure 3 : U-Net (Source : U-Net: Convolutional Networks for Biomedical Image Segmentation, Medical Image Computing and Computer-Assisted Intervention - MICCAI 2015. MICCAI 2015. Lecture Notes in Computer Science, vol 9351. Springer, Cham.)

As the idea revolved around segmentation we wanted something that can handle object detection as well as segmentation since we were looking forward to spotting an object in the image or video. Since only focusing on image segmentation was detrimental, we had to consider algorithms that are designed to handle both the tasks. R-CNN [9] did the job seamlessly. It was able to handle both tasks efficiently.

\section{R-CNN: Regions with CNN features}
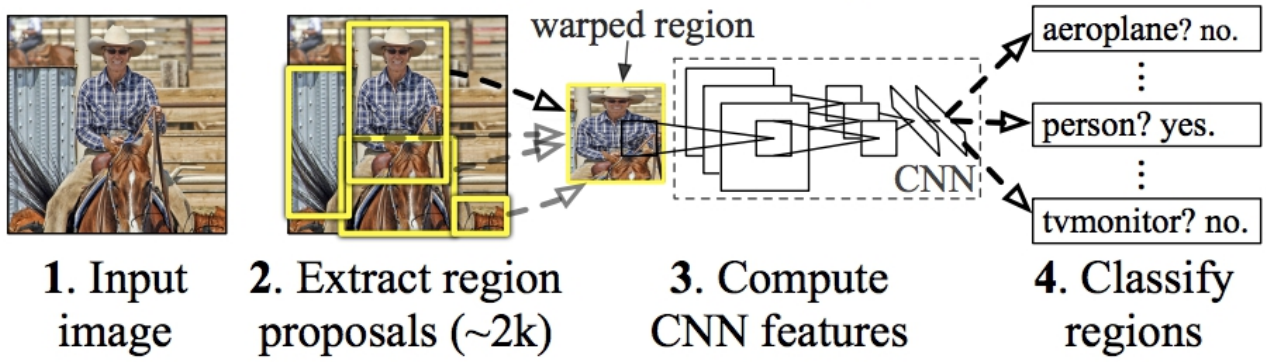
proposals $(\sim 2 \mathrm{k})$

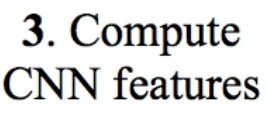
4. Classify regions

Figure 4 : R-CNN (Source : Rich feature hierarchies for accurate object detection and semantic segmentation, 2014 IEEE Conference on Computer Vision and Pattern Recognition)

We had an intuition that R-CNN would not be able to provide us everything we were looking for. The computational time required was very high. The algorithm was for sure a new start for overcoming problems but required well designed optimization for efficiency. The computational efficiency was quite poor when compared to YOLO. We wanted an algorithm that would provide computational ability as that of YOLO but get the job done. In that fashion we found an algorithm named SPP-Net which was quite better than R-CNN, but was a visual recognition algorithm and Image Segmentation statement would again digress. After a search for a while we were able to discover Fast R-CNN [10]. This was created by the same researcher who contributed towards R-CNN. The Fast R-CNN works on a multi-stage pipeline that promotes Region of Interest (RoI) [11] pooling layer. It has a truncated 
International Journal of Intelligent Communication, Computing and Networks

Open Access Journal (ISSN: 2582-7707)

https://doi.org/10.51735/ijiccn/001/21

SVD for fast detection. SVD is an abbreviation for Singular Value Decomposition [12]. It is able to train a very deep VGG-16 [13] network 9x times faster than traditional R-CNN.

Later our search came to an end with Faster R-CNN [14]. It is the fastest discovered R-CNN variant. Region Proposal Network is the principle of Faster R-CNN. The authors successfully merged Region Proposal Network (RPN) and Fast R-CNN. It continuously detects all the objects and their respective probabilities at every instance.

Later we started more intricate search as we wanted something that works in real time providing us capabilities of Image Segmentation and Object Detection. This was possible when our search came across the Yolact algorithm[15]. It was the most accurate algorithm for our problem because it was able to cover all the criterions. It produces robust high-quality masks. The prototypes for masking have been included in this network. The prototype generation also called as protonet predicts a set of $n$ prototype efficient masks for the entire input image. It was the last network we came across that was able to match our problem statement. But this is not where it is over yet.

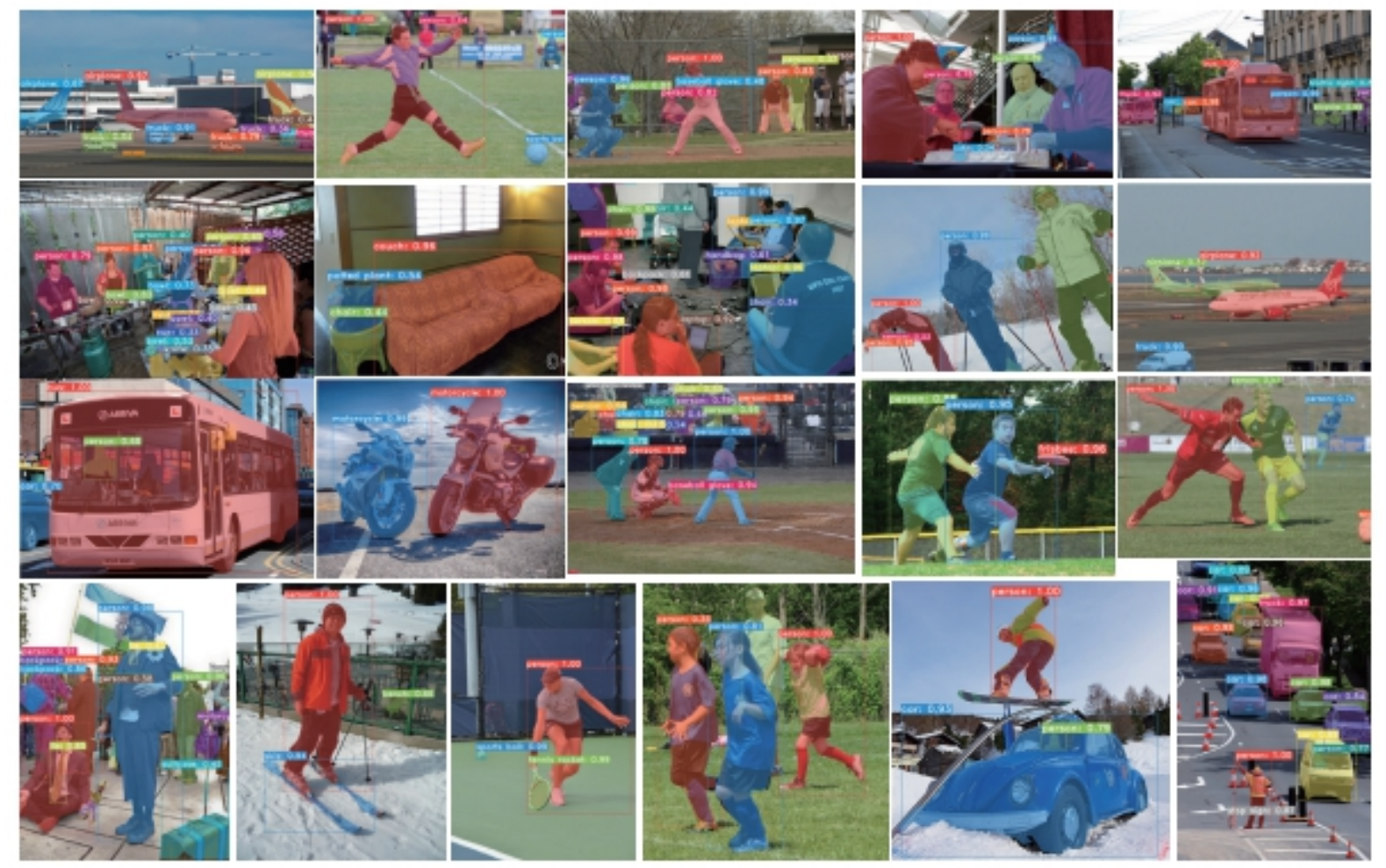

Figure 5 : Yolact (Source : YOLACT : Real-time Instance Segmentation, 2016 IEEE Conference on Computer Vision and Pattern Recognition (CVPR)

Since our search for an efficient algorithm came to halt our idea was to make manipulations in the SOTA architecture. We wanted to create the blur effect using Neural Network without being manpower induced. 


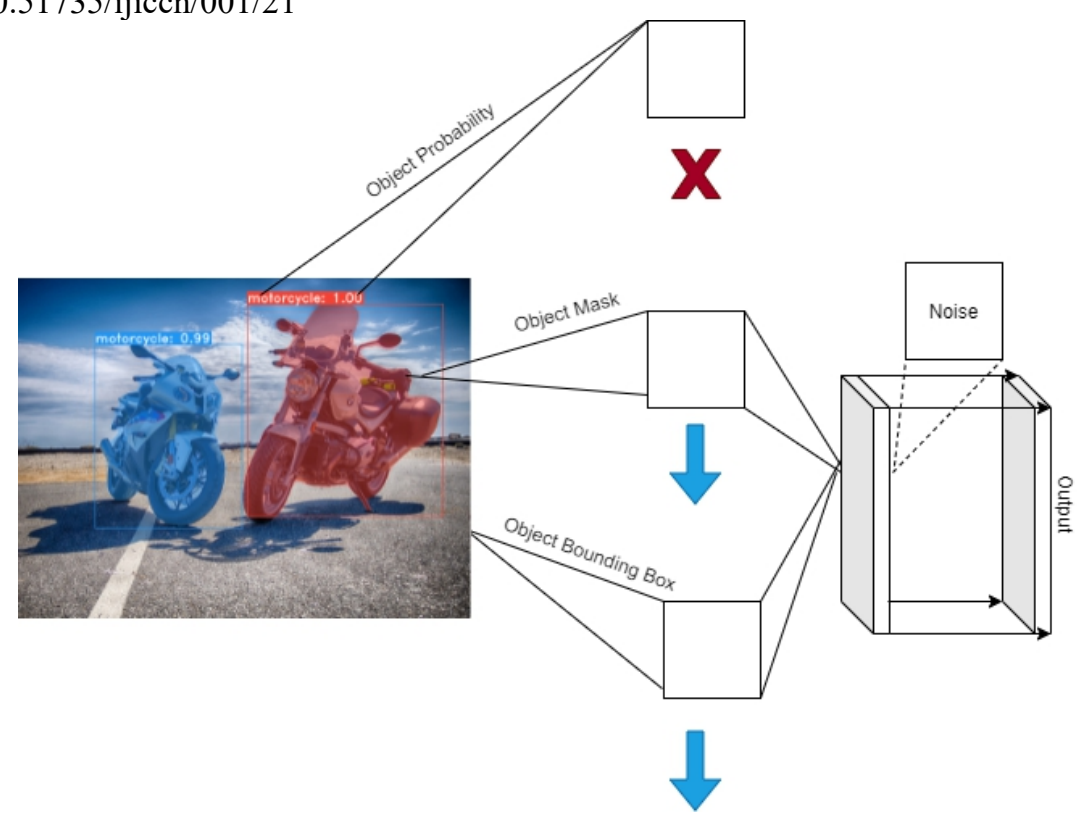

Figure 6 : The proposed system architecture after segregation and noise induction

Our proposed approach was to consider the outputs given by the Yolact. We segregated the output into 3 modules. In any prediction given by the Yolact, the components are Object Probability, Object Mask and Object Bounding Box. We wanted to eliminate the Object Probability component. Considering the other 2 components, we wanted to make reductions in them. Dimensions were the first cause. Our goal is to consider the face and reduce the dimensions accordingly. Once the dimensions are reduced the next goal is induction of Noise. A standard Noise like the Gaussian Noise [16] induction will be done to the entire Object Bounding Box. This will maintain not just throughout the mask but box too. This will be the final output layer.

\section{Discussion}

In this paper we tried to propose an idea to tackle the problem of automating blur effects for editing tools. Keeping in mind, this paper imposes a curated hypothesis of various ideologies which can help one reach the desired outcome. Our approach in this paper creates a pathway for researchers to solve the problem with images as well as videos. The idea uses Yolact with a transfer learning approach to refactor parameters and induce Gaussian Noise which is capable of replacing any heavy image/video editing tool. This idea has much more to do in future with implementation.

\section{Acknowledgments}

The idea behind the paper would have definitely not been possible without exceptional support of my friend, Mr. Mritunjay Musale (M.Tech - K. J. Somaiya College of Engineering, Mumbai). His problem spotting awareness and exacting attention to detail have been an inspiration and kept our work on track.

\section{References}

[1] Goodfellow, I.J., Bengio, Y., \& Courville, A.C. (2015). Deep Learning. Nature, 521, 436-444. 
International Journal of Intelligent Communication, Computing and Networks Open Access Journal (ISSN: 2582-7707)

https://doi.org/10.51735/ijiccn/001/21

[2] M. Mishra, \& M. Srivastava (2014). A view of the Artificial Neural Network. In 2014 International Conference on Advances in Engineering Technology Research (ICAETR - 2014) (pp. 1-3).

[3] LeCun, Y., Haffner, P., Bottou, L., \& Bengio, Y. (1999). Object Recognition with Gradient-Based Learning. Shape, Contour and Grouping in Computer Vision.

[4] Yu, S., Wickstrøm, K., Jenssen, R., \& Príncipe, J. (2021). Understanding Convolutional Neural Networks With Information Theory: An Initial Exploration. IEEE Transactions on Neural Networks and Learning Systems, 32, 435-442..

[5] Gu, J., Wang, Z., Kuen, J., Ma, L., Shahroudy, A., Shuai, B., Liu, T., Wang, X., Wang, G., Cai, J., \& Chen, T. (2018). Recent advances in convolutional neural networks. Pattern Recognit., 77, 354-377.

[6] Russakovsky, O., Deng, J., Su, H., Krause, J., Satheesh, S., Ma, S., Huang, Z., Karpathy, A., Khosla, A., Bernstein, M.S., Berg, A., \& Fei-Fei, L. (2015). ImageNet Large Scale Visual Recognition Challenge. International Journal of Computer Vision, 115, 211-252.

[7] Redmon, J., Divvala, S., Girshick, R.B., \& Farhadi, A. (2016). You Only Look Once: Unified, Real-Time Object Detection. 2016 IEEE Conference on Computer Vision and Pattern Recognition (CVPR), 779-788.

[8] Ronneberger, O., Fischer, P., \& Brox, T. (2015). U-Net: Convolutional Networks for Biomedical Image Segmentation. ArXiv, abs/1505.04597.

[9] Girshick, R.B., Donahue, J., Darrell, T., \& Malik, J. (2014). Rich Feature Hierarchies for Accurate Object Detection and Semantic Segmentation. 2014 IEEE Conference on Computer Vision and Pattern Recognition, 580-587.

[10] Girshick, R.B. (2015). Fast R-CNN. 2015 IEEE International Conference on Computer Vision (ICCV), 1440-1448.

[11] Lin, H., Si, J., \& Abousleman, G. (2007). Region-of-Interest Detection and its Application to Image Segmentation and Compression. 2007 International Conference on Integration of Knowledge Intensive Multi-Agent Systems, 306-311.

[12] Klema, V., \& Laub, A. (1980). The singular value decomposition: Its computation and some applications. IEEE Transactions on Automatic Control, 25, 164-176.

[13] Simonyan, K., \& Zisserman, A. (2015). Very Deep Convolutional Networks for Large-Scale Image Recognition. CoRR, abs/1409.1556.

[14] Ren, S., He, K., Girshick, R.B., \& Sun, J. (2015). Faster R-CNN: Towards Real-Time Object Detection with Region Proposal Networks. IEEE Transactions on Pattern Analysis and Machine Intelligence, 39, 11371149.

[15] Bolya, D., Zhou, C., Xiao, F., \& Lee, Y. (2019). YOLACT: Real-Time Instance Segmentation. 2019 IEEE/CVF International Conference on Computer Vision (ICCV), 9156-9165.

[16] Boyat, A., \& Joshi, B. (2015). A Review Paper: Noise Models in Digital Image Processing. ArXiv, abs/1505.03489. 